\title{
Alternativa Metodológica Para EI Desarrollo De La Competencia Comunicativa Oral En Idioma Inglés
}

\author{
Sandra Liliana Abarca García \\ Magíster en Enseñanza del Idioma Inglés \\ Docente de la Escuela Superior Politécnica de Chimborazo, \\ Centro de Idiomas, Ecuador \\ Maritza Lucìa Larrea Vejar \\ Diploma Superior en Metodología para la Enseñanza del Idioma Inglés. \\ Docente de la Escuela Superior Politécnica de Chimborazo, \\ Centro de Idiomas, Ecuador \\ Sandra Leticia Guijarro Paguay \\ Magister en Lingüística Aplicada al Aprendizaje del Inglés. \\ Docente de la Escuela Superior Politécnica de Chimborazo, \\ Centro de Idiomas, Ecuador
}

Doi: 10.19044/esj.2018.v14n5p160 URL:http://dx.doi.org/10.19044/esj.2018.v14n5p160

\begin{abstract}
The objective of this research, was to develop an alternative as a methodological proposal to improve the oral communicative competence in the English language, as it could determine that students do not have an adequate development of the understanding, interpretation, and recognition of vocabulary. The research is descriptive. Deductive, bibliographic and documentary methods were used. The research technique for the collection of the information was the survey while the instrument was a questionnaire validated and verified by academic peers. The research sample was intentional and represented by 20 specialists in the area of English knowledge from different institutions of higher education. The development of the proposal was made on the basis of literature review with emphasis on theoretical foundation, objectives, requirements, features, and structuring. The proposal was systematic and structured in stages, which considered objectives, actions and methodological guidance. The instrument allowed to evaluate the alternative proposal in the context of the investigation. The data obtained were tabulated, analyzed and interpreted, and represented with, charts and statistics graphics.The majority of the experts concluded, that it is adequate, structured, and it can be implemented as a methodological alternative to improve the oral communicative competence..
\end{abstract}


Keywords: Oral communicative competence, methodological alternative, English

\section{Resumen}

El objetivo de la presente investigación, fue elaborar una alternativa como propuesta metodológica para perfeccionar la competencia comunicativa oral en el idioma inglés, pues se pudo determinar, que los estudiantes no presentan un desarrollo adecuado de la comprensión, interpretación, y reconocimiento de vocabulario. La investigación es de tipo descriptiva. Los métodos aplicados fueron deductivo, bibliográfico y documental. La técnica de investigación para la recolección de la información fue la encuesta; mientras que el instrumento fue un cuestionario validado y verificado por pares académicos. La muestra fue intecionada y representada por 20 especialistas del área de conocimiento de inglés de diferentes instituciones de educación superior. La elaboración de la propuesta se realizó en base a la revisión de la literatura, con énfasis en la fundamentación, objetivos, requerimientos, características, y estructuración. La propuesta fue de aplicación sitemática y estructurada por etapas, que consideraron objetivos, acciones y orientación metodológica. El instrumento permitió evaluar la propuesta alternativa en el contexto de la investigación. Los datos obtenidos fueron tabulados, analizados e interpretados y representados con tablas y gráficos estadísticos. La mayoría de los expertos concluyeron, que es adecuada, estructurada y que puede ser implementada como una alternativa metodológica para perfeccionar la competencia comunicativa oral.

Palabras claves: Competencia comunicativa oral, Alternativa metodológica, Inglés

\section{Introducción}

Hoy más que nunca resulta imprescindible aprender el idioma Inglés. Cada día se emplea más en casi todas las áreas del conocimiento y desarrollo humano. Prácticamente puede afirmarse que se trata de la lengua del mundo actual. Es decir, en la era de la globalización, la gran lengua internacional, una "lingua franca" que ha repercutido en todos los países incluyendo a los noanglosajones, y que afecta directamente a los diversos campos y profesiones. Su posesión ya no puede tratarse como un lujo, pues se lo debe considerar como una necesidad evidente. Es más, incluso se dice ya que quien no domine esa lengua estaría en una clara situación de desventaja.

Dentro de los idiomas más estudiados en el mundo contemporáneo, está el Inglés. Quirk, R (1995), valora “... entre los parámetros fundamentales para medir la importancia de un idioma los siguientes factores: el número de habitantes nativos que tiene, en qué medida esta geográficamente extendido, 
su importancia como vehículo de comunicación y la influencia económica y política de quienes lo hablan".

Por otra parte, Edmundo W (2003), refiere que: "El Inglés es el lenguaje fundamental de libros, periódicos, aeropuertos y control del tráfico aéreo, negocios internacionales, conferencias académicas, ciencia, tecnológica, diplomacia, deportes, competencias internacionales, música popular y propaganda. Alrededor de dos tercios de científicos del mundo leen en Inglés; y al menos tres cuartos de la información electrónica almacenada están en este idioma".

Se concuerda con Deckert (1987), cuando plantea que: “... un estudiante en el que no se ha desarrollado la expresión oral puede ser debido a factores como; limitado vocabulario, falta de motivación, inclusive influye la personalidad del docente que enseña el idioma Inglés, limitado acceso a recursos didácticos, además de restringidas oportunidades de contacto con personas cuyo idioma nativo sea el Inglés entre otros".

Por otra parte el autor antes mencionado refiere que: “... otros factores que de forma evidente actúan contra la comunicación significativa y auténtica, entre los estudiantes del idioma Inglés, se encuentran según observaciones realizadas, por el habla excesiva del profesor en clase traducida en términos de comentarios y corrección frecuente, en los estudiantes, lo que conspira contra la iniciación y desarrollo de una conversación, tanto sostenida como llena de propósitos".

Con respecto al papel de la Gramática dentro del enfoque comunicativo, Jeremy Harmer en su libro The Practice of English Language Teaching establece una distinción entre gramática encubierta y gramática abierta, en dependencia de las necesidades y el nivel de los alumnos, entendiéndose por gramática encubierta aquella en la que las estructuras y funciones gramaticales no están explícitas, el estudio se centra en el texto, no en la gramática, que se absorberá inconscientemente, y es requerida preferentemente por alumnos principiantes; en tanto que la gramática abierta supone enseñar las estructuras y reglas gramaticales de forma explícita, estudiándolas y practicándolas repetidamente, y es solicitada por alumnos más avanzados.

La competencia comunicativa oral es el conjunto de técnicas que determinan las pautas generales que deben seguirse para comunicarse oralmente con efectividad, o sea, es la forma de expresar sin barreras lo que se piensa. La competencia comunicativa oral sirve como instrumento para comunicar sobre procesos u objetos externos a él, se debe tener en cuenta que en determinadas circunstancias es más amplia que el habla, ya que requiere de elementos paralingüísticos para completar su significación final. Por eso, esta no sólo implica un conocimiento adecuado del idioma, sino que abarca 
también varios elementos no verbales. La importancia de estos últimos es crucial.

Una temática que es de mucho interés en la docencia a nivel universitario es el desarrollo de la competencia comunicativa oral en idioma Inglés en el siglo XXI, es por ello que esta investigación tiene gran importancia, pues mediante la propuesta que se hace, le permitirá al estudiante poco a poco y a su ritmo, el desarrollo de la comprensión, interpretación, y reconocimiento del vocabulario técnico de su especialidad. De esta forma, el estudiante se mantendrá actualizado de lo que ocurre en el ámbito internacional, y también se apropiará de los conocimientos necesarios, en correspondencia con sus capacidades intelectuales y ha $\neg$ bilidades para su futuro desempeño profesional y social.

En la Universidad Tecnológica Equinoccial Campus Santo Domingo, objeto de investigación, el desarrollo de la competencia comunicativa oral es una de las habilidades con más insuficiencias en los estudiantes de la carrera de Comercio Exterior, a pesar de que preceden de escuelas y colegios donde han recibido clases de Inglés a lo largo de varios años, lo que equivale a muchas horas de aprendizaje de este idioma. Resulta contradictorio que al tener a los estudiantes universitarios en el aula y al empezar a trabajar con ellos en esta habilidad no la pueden hacer eficientemente, lo que origina problemas comunicativos, y esto conlleva a tener dificultades en el futuro; estos estudiantes deberían comunicarse con más fluidez en idioma Inglés. Ante esta situación se desprendieron interrogantes tales como: ¿existen diferencias significativas en el desarrollo de la competencia comunicativa en el idioma inglés entre un estudiante que tenga un buen nivel de conocimientos con otro que no lo tenga, ¿se encuentran contrastes entre estudiantes que se preocupan por incrementar su léxico para poder expresarse mejor, con otros que no aprenden nuevo vocabulario?, ¿Son adecuados los métodos de enseñanza-aprendizaje del idioma Inglés?.

A pesar de que el profesor de Inglés utiliza diferentes métodos de enseñanza-aprendizaje, existen insuficiencias en el desarrollo de la competencia comunicativa oral de los estudiantes, por ello se aplicó la presente investigación, que permitió conocer las falencias y proponer las soluciones al problema detectado en la carrera de Comercio Exterior.

Para quienes enseñan el idioma inglés, ya sea en instituciones de educación primaria, secundaria o superior o fuera de ellas, existe la preocupación en buscar una metodología que logre desarrollar eficientemente la competencia comunicativa oral.

\section{Metodología}

El enfoque de la presente investigación es de carácter descriptivo y fue aplicado a 20 especialistas o expertos procedentes de diferentes instituciones 
de Educación Superior. La investigación fue de campo, ya que se realizó para los estudiantes de la Carrera de Comercio Exterior de la Universidad Tecnológica Equinoccial, Campus Santo Domingo donde se evidencias los hechos, lo que garantizó la obtención de la información.

Es de carácter documental, pues se presenta una propuesta de solución a la problemática encontrada al contexto de la investigación.

El métodos utilizados fueron el deductivo, bibliográfico y documental, que permitió la recopilación de información a través de las técnicas de encuestas y observación.

Para alcanzar la meta de la presente investigación se definió una matriz donde se determinaron los problemas derivados que permitió establecer de forma sistémica sus fases. Por ello, en la primera fase se analizó el desarrollo histórico de la competencia comunicativa oral en idioma. Posteriormente, desde el punto de vista teórico se analizó las principales concepciones que existen en cuanto al desarrollo de la expresión oral en idioma Inglés en el nivel superior. En tercera instancia, se diagnosticó la situación actual de los estudiantes en cuanto a la comunicación oral de los estudiantes que representan la muestra. A continuación se fundamentó y elaboró la alternativa metodológica basada en el uso de los métodos problémicos para perfeccionar la competencia comunicativa oral. Finalmente, se valoró la factibilidad de aplicación de la alternativa metodológica basada en el uso de los métodos problémicos para perfeccionar la competencia comunicativa oral de los estudiantes dirigida a experto en el aréa de conocimiento de diferentes Instituciones de educación Superior.

Para la recolección de datos, se utilizó la técnica de la encuesta y observación aplicada a especialistas, la misma que fue sometida a pares académicos, quienes realizaron sus observaciones para perfeccionarla antes de ser aplicada. Como instrumentos de recolección de la información se utilizó un cuestionario que contiene la evaluación de los parámetros e indicadores que describen y aportan elementos para la elaboración de la metodología alternativa para el desarrollo de la competencia comunicativa oral del idioma inglés.

\section{Resultados}

\section{Alternativa metodológica para perfeccionar la competencia comunicativa oral en el idioma ingles, estructurada por etapas.} ETAPA I

Determinación de la situación actual de los estudiantes de la carrera de Comercio Exterior en la U.T.E. para diagnosticar la situación actual en la competencia comunicativa oral de los estudiantes de Comercio Exterior de la UTE. 
El diagnóstico se realizó en base a la técnica de preguntas y respuestas con los estudiantes.

\section{Acciones}

1. Presentación del tema objeto de análisis.

2. Entrega de un artículo relacionado a la materia.

3. Lectura rápida del artículo

4. Lectura comprensiva.

5. Revisión del vocabulario.

6. Realización de un banco de preguntas.

7. Socialización de las interrogantes.

8. Valoración de los resultados individuales.

9. Valoración de los resultados de manera grupal.

10. Socialización

En reunión convocada con los docentes de Inglés del Departamento de Idiomas se expuso los resultados obtenidos luego del diagnóstico efectuado a los estudiantes. Se receptaron los diferentes puntos de vista de los docentes acerca de las estrategias que se deben implementar para mejorar los resultados obtenidos y se procede a la selección de la técnica a diseñar para la mejora de la destreza comunicativa oral.

\section{ETAPA II}

Fundamentación metodológica para la utilización de la técnica de la discusión panel en las clases de Inglés de la carrera de Comercio Exterio, para la presentación de los fundamentos básicos en la elaboración de la técnica de discusión panel.

\section{Acciones}

1. Taller metodológico con los docentes para discutir sobre la técnica de discusión panel.

2. Presentación de los temas de las lecturas relacionadas al comercio Exterior para ser utilizadas en la técnica de discusión panel.

3. Selección de los temas relacionados a la materia de Comercio Exterior a ser considerados en discusión panel.

4. Análisis de los contenidos del programa para introducir la técnica activa de la discusión panel.

En un inicio se realizó un taller con los docentes para discutir sobre la técnica de la discusión panel, inmediatamente se presentaron los temas de las lecturas específicas de la materia de Comercio Exterior a ser utilizados, para luego análisis y seleccionar los temas de los contenidos a ser añadidos en el programa de contenidos, para finalmente presentar la propuesta. 


\section{ETAPA III}

Rediseñar el programa de Inglés a partir de la introducción de contenidos de la materia de comercio exterior en el proceso de enseñanzaaprendizaje de los estudiantes de Inglés, para diseñar la actividad a realizarse para los estudiantes de Inglés de la carrera de Comercio Exterior.

\section{Acciones}

1. Reunión con los docentes para la ubicación de los contenidos de los temas seleccionados relacionados a la materia de Comercio Exterior por cada unidad de competencia del programa.

2. Análisis metodológico de los componentes del proceso que se va a trabajar.

3. Selección del método y medio a utilizarse en la implementación de la alternativa metodológica.

4. Realización del bosquejo de las actividades a ejecutarse en reunión con los docentes.

Como primer paso en la reunión con los docentes se ubicó el listado de temas en cada unidad de competencia del programa, a fin de analizar los componentes del proceso metodológico con el que se va a trabajar. A continuación se seleccionó el método y el medio a emplear para implementar la estrategia metodológica, para concluir en conjunto con los docentes, se diseña un bosquejo de las actividades a ejecutarse.

\section{ETAPA IV}

Ejemplificación del tratamiento metodológico del uso de la técnica de la discusión panel en una clase de Inglés de la carrera de comercio exterior para demostrar a través de una clase de inglés con los estudiantes de Comercio Exterior como desarrollar la actividad de la discusión panel.

\section{Acciones}

Moderar la clase:

A continuación se presenta la clase:

1. Con los estudiantes se aplica una técnica de animación para crear un ambiente fraterno y de confianza.

2. Se realiza un ejercicio encaminado a conocer las expectativas que tienen los estudiantes de Comercio Exterior del idioma inglés con respecto a la competencia comunicativa oral.

3. Se presenta los lineamientos de la actividad en clase para la realización de la técnica de la discusión panel.

4. Se da a conocer a los estudiantes sobre los parámetros de la evaluación.

5. Se pone en práctica la técnica con los estudiantes. 
Se inició con la aplicación de una técnica de animación para crear un ambiente fraterno y de confianza entre los estudiantes, para posteriormente realizar un ejercicio encaminado a conocer las expectativas que tienen los estudiantes de Comercio Exterior del idioma Inglés con respecto a la competencia comunicativa oral, a continuación se presentó los lineamentos de la actividad en clase para la realización de la técnica de discusión panel. Se continuó con dar a conocer a los estudiantes sobre los parámetros de la evaluación y finalmente se puso en práctica la técnica con los estudiantes.

Las próximas clases fueron de ejercitación se debe tener en cuenta el nivel de conocimiento de los participantes. Fue importante manteber en la clase de Inglés un estado de satisfacción y alegría durante el desarrollo de las actividades propuestas.

\section{Validación de propuesta}

Se consideró un total de 20 especialistas o expertos procedentes de diferentes instituciones de Educación Superior, a quienes se les aplicó el instrumento de recolección de la información, los cuales manifiestaron sus criterios.

Pregunta 1. ¿Cómo evalúa la fundamentación de la propuesta fundamentación de la propuesta metodológica para el desarrollo de la competencia comunicativa oral en idioma inglés?

Tabla 1. Fundamentación de la propuesta metodológica para el desarrollo de la competencia comunicativa oral en idioma inglés

\begin{tabular}{cccccc}
\hline Aspectos & $\begin{array}{c}\text { Muy } \\
\text { adecuada }\end{array}$ & $\begin{array}{c}\text { Bastante } \\
\text { adecuada }\end{array}$ & Adecuada & $\begin{array}{c}\text { Poco } \\
\text { adecuada }\end{array}$ & No adecuda \\
\hline Fundamentación & 18 & 2 & 0 & 0 & 0 \\
\hline
\end{tabular}

Fuente: Especialistas en lengua inglesa

Elaborado por: Abarca Sandra

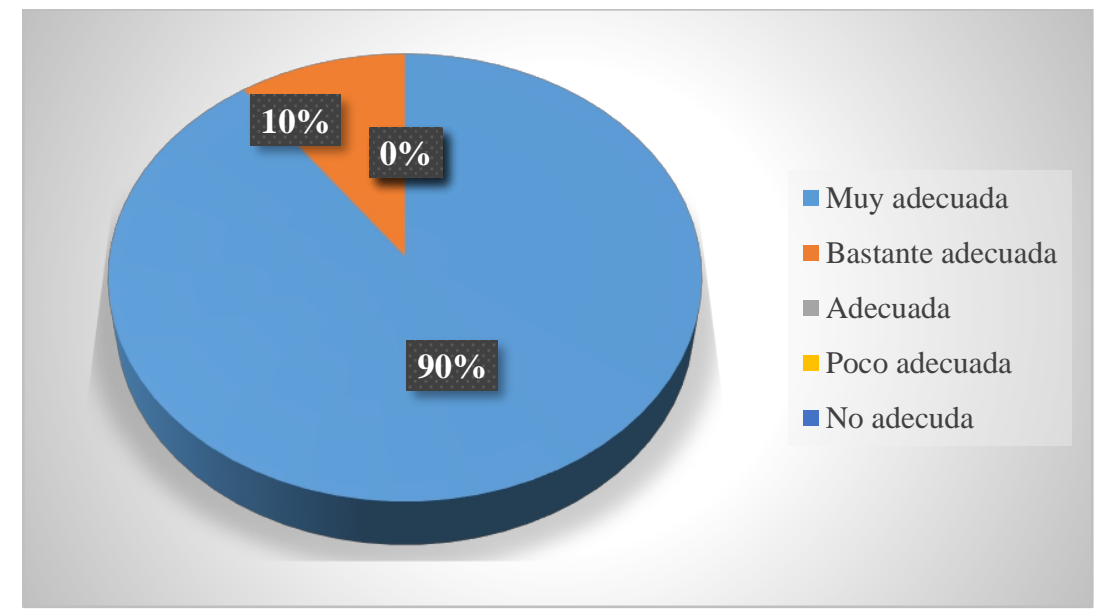

Gràfico 1. Fundamentación de la propuesta metodológica para el desarrollo de la competencia comunicativa oral en idioma inglés. 


\section{Análisis e interpretación}

Del $100 \%$ de especialistas o expertos procedentes de diferentes instituciones de Educación Superior, el 90\% considera Muy adecuada la fundamentación de la propuesta metodológica, el 10\% Bastante adecuada; mientras no se registran frecuencias en las restantes opciones. Es decir, que la mayoría de los especialitas considera positiva la fundamentación de la propuesta y no contiene argumentaciones para la metodológica para el desarrollo de la competencia comunicativa oral en idioma inglés.

Pregunta 2. ¿Cómo considera usted el objetivo de la propuesta metodológica para el desarrollo de la competencia comunicativa oral en idioma inglés?

Tabla 2. Objetivo de la propuesta metodológica para el desarrollo de la competencia comunicativa oral en idioma inglés

\begin{tabular}{cccccc}
\hline Aspectos & $\begin{array}{c}\text { Muy } \\
\text { adecuada }\end{array}$ & $\begin{array}{c}\text { Bastante } \\
\text { adecuada }\end{array}$ & Adecuada & $\begin{array}{c}\text { Poco } \\
\text { adecuada }\end{array}$ & No adecuda \\
\hline Objetivo & 16 & 2 & 2 & 0 & 0 \\
\hline
\end{tabular}

Fuente: Especialistas en lengua inglesa

Elaborado por: Abarca Sandra

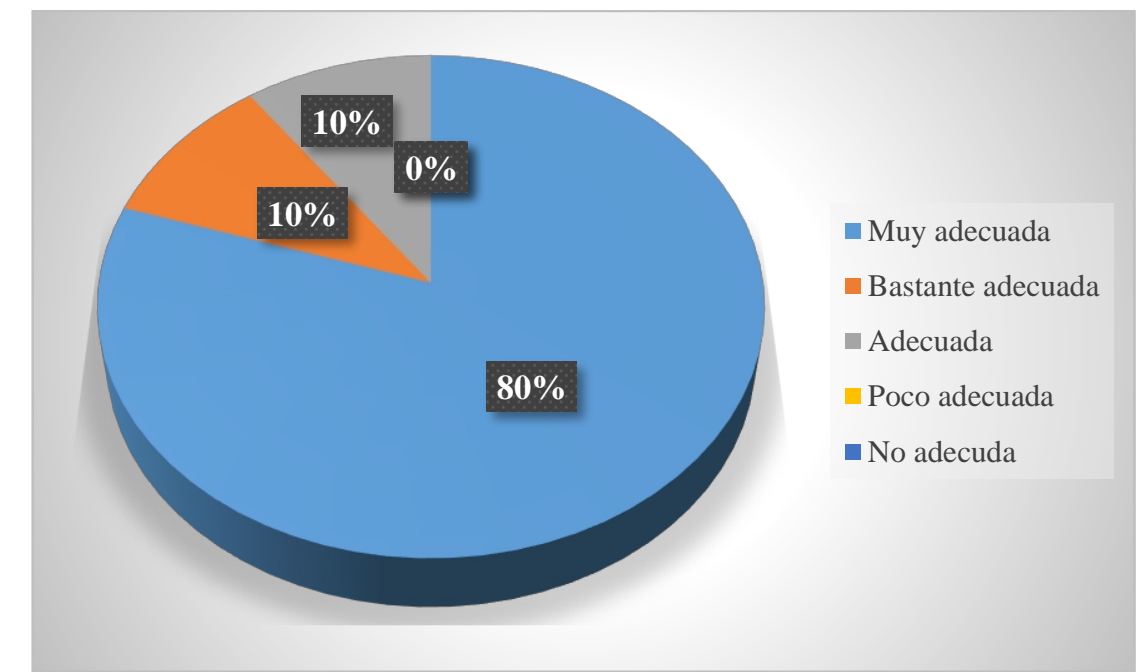

Gráfico 2. Objetivo de la propuesta metodológica para el desarrollo de la competencia comunicativa oral en idioma inglés

\section{Análisis e interpretación}

Del $100 \%$ de especialistas o expertos procedentes de diferentes instituciones de Educación Superior, el 80\% considera Muy adecuada el objetivo de la propuesta metodológica, el 10\% Bastante adecuada y Adecuada; mientras no se registran frecuencias en las restantes opciones. Es decir, que la mayoría de los especialitas considera.

La mayoría de especialistas aprueba los objetivos de la propuesta, por lo que se podrán alcanzar las metas establecidas en la metodológica para el desarrollo de la competencia comunicativa oral en idioma inglés. 
Pregunta 3. ¿Los requerimientos están bien planteados o usted propondría otros en la propuesta metodológica para el desarrollo de la competencia comunicativa oral en idioma inglés?

Tabla 3. Requerimiento planteados en la propuesta metodológica para el desarrollo de la competencia comunicativa oral en idioma inglés

\begin{tabular}{cccccc}
\hline Aspectos & $\begin{array}{c}\text { Muy } \\
\text { adecuada }\end{array}$ & $\begin{array}{c}\text { Bastante } \\
\text { adecuada }\end{array}$ & Adecuada & $\begin{array}{c}\text { Poco } \\
\text { adecuada }\end{array}$ & No adecuda \\
\hline Requerimientos & 18 & 1 & 1 & 0 & 0 \\
\hline \multicolumn{5}{c}{ Fuente: Especialistas en lengua inglesa } \\
Elaborado por: Abarca Sandra
\end{tabular}

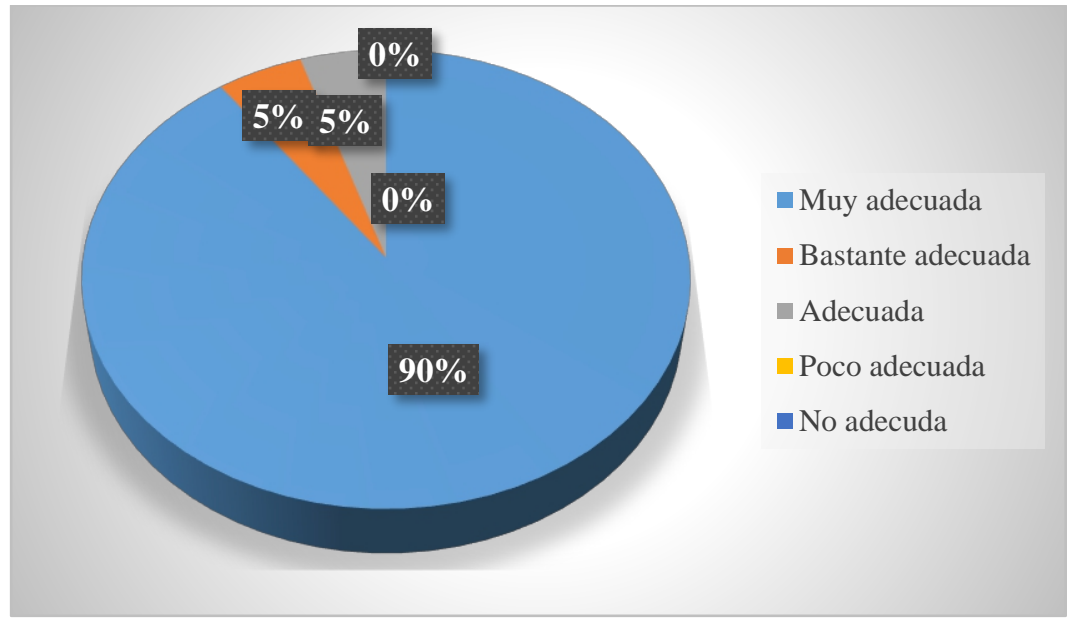

Gráfico 3. Requerimiento planteados en la propuesta metodológica para el desarrollo de la competencia comunicativa oral en idioma inglés

\section{Análisis e interpretación}

Del $100 \%$ de especialistas o expertos procedentes de diferentes instituciones de Educación Superior, el 90\% considera Muy adecuada los requemientos de la propuesta metodológica, el 5\% Bastante adecuada y Adecuada; mientras no se registran frecuencias en las restantes opciones.

La mayoría de especialistas sostienen que se ha considerado todos los requerimientos en el planteamiento de la propuesta metodológica para el desarrollo de la competencia comunicativa oral en idioma inglés.

Pregunta 4. ¿Qué opina sobre las características mencionadas en esta propuesta metodológica para el desarrollo de la competencia comunicativa oral en idioma inglés?

Tabla 4. Características planteados en la propuesta metodológica para el desarrollo de la competencia comunicativa oral en idioma inglés

\begin{tabular}{cccccc}
\hline Aspectos & $\begin{array}{c}\text { Muy } \\
\text { adecuada }\end{array}$ & $\begin{array}{c}\text { Bastante } \\
\text { adecuada }\end{array}$ & Adecuada & $\begin{array}{c}\text { Poco } \\
\text { adecuada }\end{array}$ & No adecuda \\
\hline Características & 15 & 3 & 1 & 1 & 0 \\
\hline
\end{tabular}

Fuente: Especialistas en lengua inglesa

Elaborado por: Abarca Sandra 


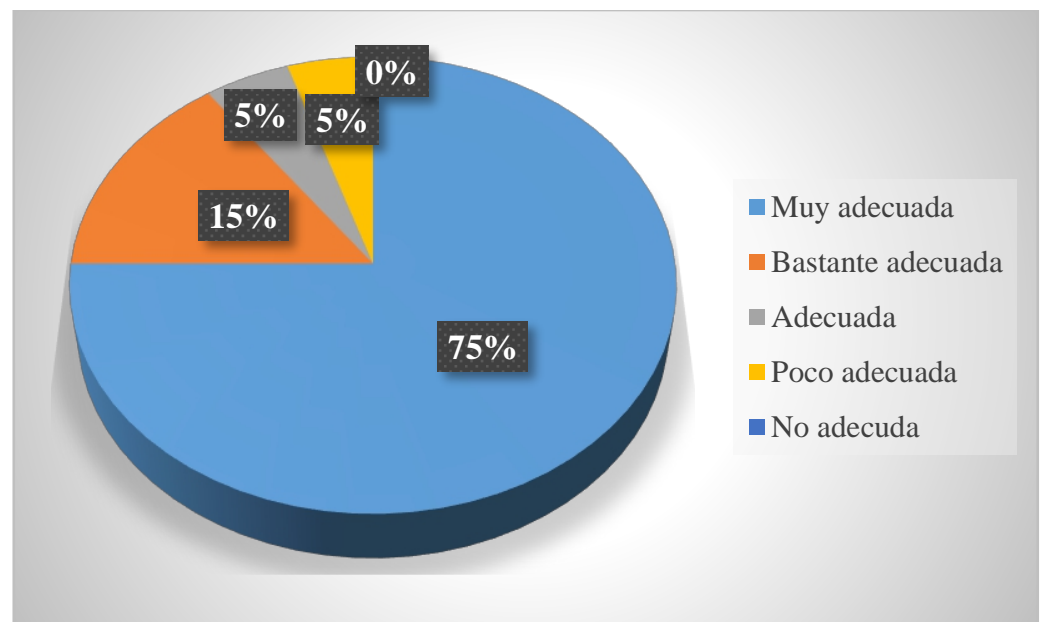

Gráfico 4. Características planteados en la propuesta metodológica para el desarrollo de la competencia comunicativa oral en idioma inglés

\section{Análisis e interpretación}

Del $100 \%$ de especialistas o expertos procedentes de diferentes instituciones de Educación Superior, el 75\% considera Muy adecuada los características de la propuesta metodológica, el 15\% Bastante adecuadas, el 5\% Adecuada y Poco adecuadas; mientras no se registran frecuencias en las restantes opciones.

La mayoría de especialistas considera que se han contenplados las debidas características en el planteamiento de la propuesta metodológica para el desarrollo de la competencia comunicativa oral en idioma inglés, un pequeño procentaje, sostiene que son poco adecuadas.

Pregunta 5. ¿Considera oportuno las etapas planteados en la propuesta metodológica para el desarrollo de la competencia comunicativa oral en idioma inglés?

Tabla 4. Etapas planteadas en planteados en la propuesta metodológica para el desarrollo de la competencia comunicativa oral en idioma inglés

\begin{tabular}{cccccc}
\hline Aspectos & $\begin{array}{c}\text { Muy } \\
\text { adecuada }\end{array}$ & $\begin{array}{c}\text { Bastante } \\
\text { adecuada }\end{array}$ & Adecuada & $\begin{array}{c}\text { Poco } \\
\text { adecuada }\end{array}$ & No adecuda \\
\hline Etapas & 20 & 0 & 0 & 0 & 0 \\
\hline
\end{tabular}

Fuente: Especialistas en lengua inglesa

Elaborado por: Abarca Sandra 


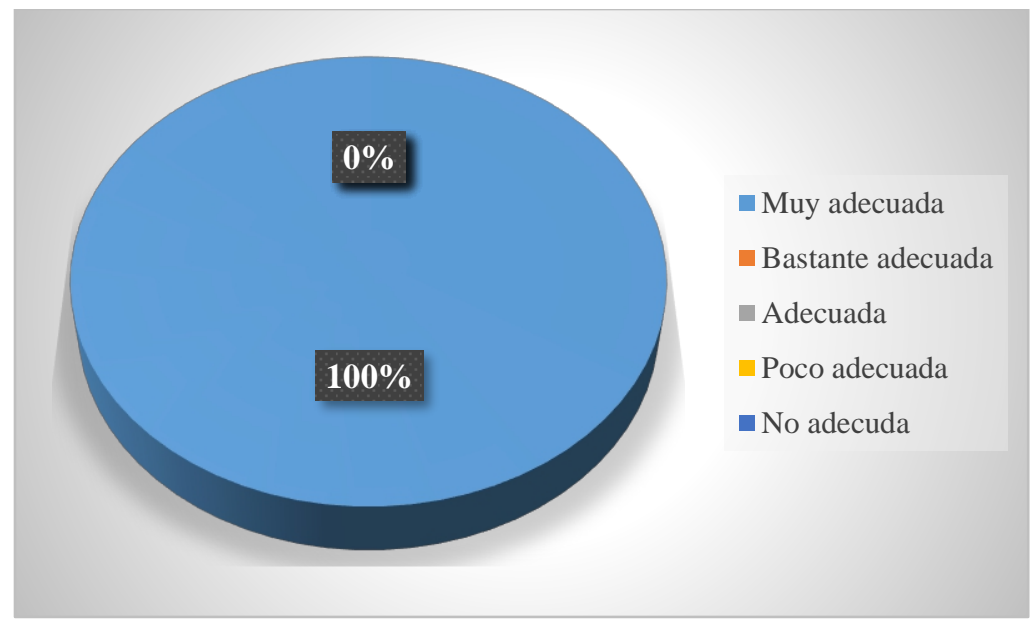

Gráfico 4. Etapas planteadas en planteados en la propuesta metodológica para el desarrollo de la competencia comunicativa oral en idioma inglés.

\section{Análisis e interpretación}

Del $100 \%$ de especialistas o expertos procedentes de diferentes instituciones de Educación Superior, el 100\% considera Muy adecuada las etapas de la propuesta metodológica; mientras no se registran frecuencias en las restantes opciones. Es decir que la totalidad de especialistas están de acuerdo con las etapas planteadas.

\section{Conclusion}

Desde el punto de vista histórico, y con el análisis desarrollado en el transcurso investigativo del proceso de enseñanza - aprendizaje del inglés. Se confirmó que ha evolucionado de un enfoque tradicionalista a un enfoque desarrollador y comunicativo en lo que concierne a la competencia comunicativa oral, a pesar de que en muchas ocasiones se centra en actividades de carácter general que no favorecen el desarrollo de la competencia comunicativa oral.

La valoración de los principales referentes teóricos sobre el proceso de enseñanza aprendizaje permitió corroborar la necesidad que existe de argumentar desde una nueva perspectiva teórica y metodológica el desarrollo de la competencia comunicativa oral a nivel superior.

De la misma manera la competencia comunicativa oral no se ha perfeccionado porque en un gran porcentaje el docente de inglés no utiliza una metodología apropiada teniendo una menor importancia el uso de técnicas activas de enseñanza aprendizaje.

Se elaboró una estrategia metodológica que tiene como característica el uso de la técnica activa de la discusión panel que permite el desarrollo de la competencia comunicativa oral en los estudiantes de la carrera de Comercio 
Exterior, lo que posibilita la pertinencia de la investigación al revertirse en los estudiantes del nivel superior objeto de nuestro estudio.

De acuerdo al criterio de los expertos considieron que los parámetros y las etapas previstas para la aplicación de la alternativa metodológica para el desarrollo de la competencia comunicativa oral en idioma inglés son los adecuados.

\section{References:}

1. Antich, de León R., Gandarias, Cruz D., y López, Segrera, E. (1986). Metodología de la Enseñanza de Lenguas Extranjeras. Teoría de la Educación en Pedagogía. Editorial Pueblo y Educación. La Habana, 1986. (p. 49-59)

2. Canale, M., \& Swain, M. (1980). Theoretical bases of communicative approaches to second Caracas. Universidad Simón Bolívar.

3. Deckert, G. (1987). La competencia comunicativa y su relación con la enseñanza del idioma inglés en las" Ciencias Biomédicas"

4. Harmer, Jeremy. (2001). The Practice of English Language Teaching. England: Pearson Education Limited, Third Edition.

5. Quirk R., et al. (1985). A Comprehensive Grammar of the English Language.

6. Richards, J. C., Platt, J. Y Platt, H. (1985) Longman dictionary of language teaching and applied linguistics, Londres, Longman.

7. Quirk R., et al. (1985). A Comprehensive Grammar of the English Language. London, Longman. 